\title{
Minimization of rate of inbreeding for small populations with overlapping generations
}

\author{
A. K. SONESSON* AND T. H. E. MEUWISSEN \\ ID-Lelystad, Institute for Animal Science and Health, PO Box 65, 8200 AB Lelystad, The Netherlands
}

(Received 26 June 2000 and in revised form 9 November 2000 and 22 January 2001)

\begin{abstract}
Summary
We propose a method that minimizes the rate of inbreeding $(\Delta F)$ for small unselected populations with overlapping generations and several reproductive age classes. It minimizes the increase in coancestry of parents and optimizes the contribution of each selection candidate. The carrying capacity of the population is limited to a fixed number of animals per year. When survival rate equalled $100 \%$, only animals from the oldest age class were selected, which maximized the number of parents per generation, slowed down the turnover of generations and minimized the increase of coancestry across sublines. However, the population became split into sublines separated by age classes, which substantially increased inbreeding within sublines. Sublines were prevented by a restriction of selecting at least one sire and one dam from the second-oldest age class, which resulted in an $L$ times lower $\Delta F$, where $L$ equals the average generation interval of sires and dams. Minimum coancestry mating resulted in lower levels of inbreeding than random mating, but $\Delta F$ was approximately the same. For schemes where the oldest animals were selected, $\Delta F$ increased by $18-52 \%$ compared with the proposed method.
\end{abstract}

\section{Introduction}

For small and endangered populations, it is important to avoid inbreeding as much as possible, because increased inbreeding leads to inbreeding depression, lower genetic variance and a higher frequency of lethal genes. When the breeding of the population can be controlled, there are several methods to reduce or minimize the long-term rate of inbreeding, $\Delta F$. One method equalizes family size (EFS) (Wright, 1931; Wang, 1997a). Recently, an extreme form of EFS was proposed by Wang \& Hill (2000) that used markerassisted selection (MAS) to reduce the variance of each contributed allele. In principle, the effective population size could increase infinitely with MAS, but in practice the amount of marker information, the genome size and the number of marker-genotyped offspring per family is limiting. Another method is the minimization of coancestry selection, where animals are selected such that the average coancestry of parents is minimized. This method is more practical

\footnotetext{
* Corresponding author. Tel: + 31 (0)320 238238. Fax : + 31 (0) 320 238050. e-mail: a.k.sonesson@id.wag-ur.nl.
}

than EFS, because it can handle temporary distortion of family sizes due, for example, to involuntary culling of animals. Finally, the average coancestry of mated animals and thus also the inbreeding level of the next generation can be minimized by minimum coancestry (MC) mating.

The above selection and mating methods minimize inbreeding for populations with discrete generations. In practice, however, generations are overlapping with complex pedigrees and high variance of family size for most species (Ballou \& Lacy, 1995). For schemes with overlapping generations, there are selection candidates from several reproductive age classes and an inbreeding minimization method has to account for earlier (unequal) contributions of parents. For overlapping generation structures, Wang et al. (1994) tested several heuristic selection indices, which mainly showed that prolonged generation intervals are effective in reducing $\Delta F$.

Robertson (1964) concluded that the overall rate of inbreeding would be minimized if the population was split into sublines and there was some mixing of the sublines. Within the sublines, inbreeding and genetic drift would be increased, but genetic drift of the entire 
population would be decreased in the long term. This becomes more apparent if we consider a single biallelic locus with alleles $A$ and $a$. In a single population, one of the alleles $A$ or $a$ is eventually lost, whereas in some sublines the $A$ allele will become fixed while in others it will be the $a$ allele, i.e. the subline structure will maintain both alleles. However, in practice, the sublines may become so highly inbred that they do not survive the inbreeding depression (Wang \& Hill, 2000).

For random mating, Wray \& Thompson (1990) showed that the variance of long-term contributions from ancestors to descendants, $S_{\mathrm{c}}^{2}$, is related to the rate of inbreeding as $\Delta F=\left(1+S_{\mathrm{c}}^{2}\right) / 4 N$, where $N$ is the number of breeding animals per generation (assumed to be constant over generations). Because $S_{\mathrm{c}}^{2}$ equals zero when all ancestors have equal contributions to the descendants, rates of inbreeding are then minimized. Woolliams \& Thompson (1994) extended the above expression to account for non-random (e.g. MC mating or sublining):

$\Delta F=\left(1+S_{\mathrm{c}}^{2}\right)(1-\alpha) / 4 N$,

where $\alpha$ is the correlation of genes within individuals. For MC mating, where animals with least similar genes are mated, animals will show a negative correlation between their genes $(\alpha<0)$. Therefore, $\Delta F$ can actually be increased by MC mating, i.e. the term $(1-\alpha)>1$. However, MC mating also connects unrelated families with each other, such that the genetic contributions become more equal, i.e. it reduces $S_{\mathrm{c}}^{2}$ and therefore also $\Delta F$ (Caballero \& Toro, 2000).

The aim of this paper is to develop a method that minimizes inbreeding for small populations with overlapping generations and complex pedigrees. We test the effects of minimum coancestry selection and MC mating on inbreeding for overlapping generation structures. We consider a situation where the number of animals born per time period limits the population size. In practice, all mature females of endangered breeds will be mated, but since the carrying capacity of the local captive population is limited, the offspring from some females will be sold, moved to other populations or released into wild populations.

\section{Materials and methods}

(i) Minimization of coancestry selection for overlapping generations ( $\mathrm{MCO}$ )

The objective is to minimize the average coancestry of parents. We want to select parents and set up matings for the $N$ newborn animals in the population of the next year. The problem is to find an optimum, c, which minimizes $\mathbf{c}^{\prime} \mathbf{A c}$ and thus also $\frac{1}{2} \mathbf{c}^{\prime} \mathbf{A c}$, where $\mathbf{c}$ is the contribution for each selection candidate and $\mathbf{A}$ is an $(n \times n)$ matrix with relationship coefficients between selection candidates, which equal 2 times the coancestry coefficients (Meuwissen, 1997). For schemes with overlapping generations, animals are assumed to be in discrete yearly age classes, but other time units can also be used. Animals up to and including the oldest reproductive age have the potential to carry genes of future generations, so young age classes that are not yet reproducing have also to be considered. The average relationships in a population with overlapping generations are obtained by weighting the average relationships within and between age classes by the long-term contribution of the age classes $(r)$, which is obtained from Hill's (1974) geneflow method (Meuwissen \& Sonesson, 1998):

$r_{1}^{2} \mathbf{c}^{\prime} \mathbf{A} \mathbf{c}+2 r_{1} \mathbf{c}^{\prime} \mathbf{A} \mathbf{J} \mathbf{r}_{2}+\mathbf{r}_{2}^{\prime} \overline{\mathbf{A}}_{22} \mathbf{r}_{2}$,

where subscripts 1 and 2 denote the youngest age class (new progeny) and older age classes respectively $\left(r_{1}=\right.$ long-term contributions of new progeny age class from Hill's (1974) gene-flow method, $\mathbf{r}_{2}=((q-1) \times 1)$ vector of long-term contributions of older age classes and $q=$ total number of age classes), $\quad \overline{\mathbf{A}}_{22}=$ $(q-1) \times(q-1)$ matrix of average relationships between older age classes. The $\left(\mathbf{c}^{\prime} \mathbf{A c}\right)$ term equals the average relationship between new progeny, the $\left(\mathbf{c}^{\prime} \mathbf{A} \mathbf{J} \mathbf{r}_{2}\right)$ term equals the relationship between old animals and new progeny and the term $\left(\mathbf{r}_{2}^{\prime} \overline{\mathbf{A}}_{22} \mathbf{r}_{2}\right)$ equals average relationships between old animals. The $\mathbf{J}$ matrix is an $(n \times(q-1))$ matrix that averages individual relationship to average relationship per age class, i.e. $J_{i j}=1 / m_{j}$ if animal $i$ belongs to age class $j$, and $J_{i j}=0$ otherwise, where $m_{j}$ is the number of animals in age class $j$. The relationships between older animals $\left(\mathbf{r}_{2}^{\prime} \overline{\mathbf{A}}_{22} \mathbf{r}_{2}\right)$ are not affected by the current selection, because relationships between old animals are already established.

Because the genetic contributions of all males and of all females must sum to $\frac{1}{2}$, a restriction is added:

$\mathbf{Q}^{\prime} \mathbf{c}=\frac{1}{2} \mathbf{1}_{2}$,

where $\mathbf{Q}$ is a known incidence matrix for sex; the first column yields ones for males and zeros for females and the second column yields ones for females and zeros for males; $\mathbf{1}_{\mathbf{2}}$ is a $(2 \times 1)$ vector of ones. In the Appendix, (2) is minimized under the restriction (3), giving:

$\mathbf{c}=\mathbf{A}^{-1} \mathbf{Q} \lambda / 2 r_{1}^{2}-\mathbf{J r}_{2} / r_{1}$,

where $\lambda$ equals

$\lambda=2 r_{1}\left(\mathbf{Q}^{\prime} \mathbf{A}^{-1} \mathbf{Q}\right)^{-1}\left(r_{1} \mathbf{s}+\mathbf{Q}^{\prime} \mathbf{J} \mathbf{r}_{2}\right)$.

In the previous section, the vector of long-term contributions, $\mathbf{r}$, was assumed known. The vector is 
calculated from the gene-flow matrix between age classes, $\mathbf{P}$, and depends on the contribution of each age class to age class 1 (Hill, 1974). These contributions depend, however, on the age of selected animals, i.e. on c. Thus, iteration is needed to find the optimal c and $\mathbf{r}$ simultaneously (Meuwissen \& Sonesson, 1998). Alternatively, simulated annealing could be used to calculate the optimum $\mathbf{r}$ (Grundy et al., 1998). Both algorithms yielded very similar results on selection schemes (Sonesson et al., 2000). The iteration algorithm is used here, because it requires less computer time.

(ii) Mating schemes

\section{(a) Minimum coancestry (MC) mating}

Minimum coancestry seems a useful objective to determine the mating pairs, since it minimizes the inbreeding coefficients of the offspring and therefore avoids inbreeding depression as much as possible for the next generation (Wright, 1921). MC mating will be used here in combination with MCO selection schemes.

To find the mating structure that yields the minimum coancestry among the mating pairs, a matrix F of size $\left(N_{\mathrm{s}} \times N_{\mathrm{d}}\right)$ is set up, where $N_{\mathrm{s}}\left(N_{\mathrm{d}}\right)$ is the number of selected sires (dams) and element $\mathbf{F}_{\mathbf{i j}}$ is the coefficient of coancestry of the selected individuals $i$ and $j$, which is also the inbreeding coefficient of their progeny. The number of matings for each animal is obtained from its optimum contribution, $\mathbf{c}$, and is calculated by the selection algorithm by multiplying the contributions of the sire (dam) by the number of selection candidates and rounding to integers. Normally, the truncation point for rounding up versus down is 0.5 , but if the total number of progeny does not sum to the intended number, the truncation point is adjusted such that the intended number of progeny results. This is not guaranteed to give the optimum integer solution, but probably yields a reasonable approximation.

The MC mating structure is obtained using the simulated annealing algorithm (Press et al., 1989). The implementation of the annealing algorithm is described in the appendix of Sonesson \& Meuwissen (2000).

\section{(b) Random (RAND) mating}

For the random mating schemes (RAND), the simulated annealing algorithm is used as explained in the MC mating section above, but all changes were accepted and the total number of random changes was set to 10000 . In this way, the number of progeny per sire (dam) was restricted to the numbers defined by MCO, while still achieving random mating (Sonesson \& Meuwissen, 2000). In practice, simpler algorithms can be devised to achieve this, but note that simply sampling sires and dams according to the proportions defined by the $\mathbf{c}$ vector results in random deviations from the number of offspring defined by MCO.

\section{(iii) Simulated schemes}

For overlapping generation schemes, a closed nucleus scheme was simulated for 100 years. Schemes were symmetric with respect to the two sexes and there were no animals with limited reproductive capacity. One hundred replicated simulations were obtained for each scheme.

For convenience, it was assumed that one year equals one age class. Animals started to reproduce at age 1 , continuing until the oldest reproductive age 9, resulting in nine reproductive age classes. Survival rate was $70 \%$, which means that each individual has a probability of $70 \%$ of surviving every selection round (year). Although a survival rate of $100 \%$ is not realistic, schemes with $100 \%$ survival were also simulated in order to test whether the algorithm would create sublines of age classes. There were four reproductive age classes (1,2,3 and 4) for schemes with $100 \%$ survival. The variance of contributions of ancestors to descendants, $S_{\mathrm{c}}^{2}$, was obtained by defining an entire generation of ancestors as a consecutively born group of animals whose long-term contributions sum to 1 (Bijma \& Woolliams, 1999). The correlation of genes within an animal, $\alpha$, was derived from Wright's (1969) $F$-statistics using

$(1-F)=(1-\alpha)(1-k)$,

where $F$ is the inbreeding of offspring (Wright's $F_{\text {IT }}$ ); $k$ is the average kinship among parents (equals inbreeding of offspring with random mating; Wright's $\left.F_{\mathrm{ST}}\right)$ and $\alpha$ denotes a deviation of inbreeding due to non-random mating (Wright's $F_{\text {IS }}$ ).

The $\Delta F$ and increase of coancestry, $\Delta C$, were calculated as an average over years 96-100. For overlapping generations and $70 \%$ or $100 \%$ survival, RAND and MC were compared for 6,10 or 20 newborn animals per year.

\section{Results}

(i) $100 \%$ survival

For schemes with overlapping generations and $100 \%$ survival, the maximum generation interval was 4 years and four age classes (1,2,3 and 4) were eligible for selection. For both RAND and MC mating, MCO selected only the oldest animals (Table 1), which 
Table 1. Rate of inbreeding per year $(\Delta F)$, increase in coancestry per year $(\Delta C)$, generation interval of sires/dams $(L)$ number of selected sires/dams $(N \mathrm{sel})$ and number of male/female selection candidates (Ncand) for schemes with 6,10, and 20 newborn animals per year and $100 \%$ survival

\begin{tabular}{llllll}
\hline \hline Mating method $^{a}$ & $\Delta F^{b}$ & $\Delta C^{b}$ & $L$ (years) & $N$ sel & $N$ cand \\
\hline Six animals & & & & & \\
RAND & $0 \cdot 0117$ & $0 \cdot 0119$ & $4 \cdot 0 / 4 \cdot 0$ & $3 \cdot 0 / 3 \cdot 0$ & $12 / 12$ \\
MC & $0 \cdot 0120$ & $0 \cdot 0123$ & $4 \cdot 0 / 4 \cdot 0$ & $3 \cdot 0 / 3 \cdot 0$ & $12 / 12$ \\
Ten animals & & & & & \\
RAND & $0 \cdot 0072$ & $0 \cdot 0067$ & $4 \cdot 0 / 4 \cdot 0$ & $5 \cdot 0 / 5 \cdot 0$ & $20 / 20$ \\
MC & $0 \cdot 0069$ & $0 \cdot 0070$ & $4 \cdot 0 / 4 \cdot 0$ & $5 \cdot 0 / 5 \cdot 0$ & $20 / 20$ \\
Twenty animals & & & & & \\
RAND & $0 \cdot 0033$ & $0 \cdot 0032$ & $4 \cdot 0 / 4 \cdot 0$ & $10 \cdot 0 / 10 \cdot 0$ & $40 / 40$ \\
MC & $0 \cdot 0034$ & $0 \cdot 0033$ & $4 \cdot 0 / 4 \cdot 0$ & $10 \cdot 0 / 10 \cdot 0$ & $40 / 40$ \\
\hline \hline
\end{tabular}

${ }^{a}$ RAND, random mating; MC, minimum coancestry mating.

${ }^{b}$ Average of years 96-100; standard errors are between 0.0000 and 0.0020.

Table 2. An illustration of the occurrence of sublining in a breeding scheme where all animals have a generation interval of 4 years. The four sublines are denoted $A, B, C$ and $D$ and are separated by time

\begin{tabular}{lllll}
\hline \hline \multicolumn{5}{c}{ Age classes } \\
Year & 1 & 2 & 3 & 4 \\
\cline { 2 - 5 } 1 & A & B & C & D \\
2 & D & A & B & C \\
3 & C & D & A & B \\
4 & B & C & D & A \\
5 & A & B & C & D \\
$\ldots$ & $\cdots$ & $\cdots$ & $\cdots$ & $\cdots$ \\
\hline \hline
\end{tabular}

a When going from one year to the next, sublines in age classes 1,2 and 3 became 1 year older and the subline in age class 4 reproduces to obtain offspring in age class 1 of the next year.

maximizes the number of parents per generation and slows down the turnover of generations. However, it also results in complete sublining of age classes as in the example of Table 2. Note that complete sublining requires that all parents come from the same age class (age class 4 in Table 2), which results in a small number of parents when few parents survive up to this age class. Hence, a survival rate of $100 \%$ maximizes the probability of sublining. Complete sublining resulted in high $\Delta F(0.0072$ and 0.0069 for schemes with 10 newborn animals per year and RAND and MC mating, respectively) and $\Delta C(0.0067$ and 0.0070 per year for RAND and MC mating, respectively), because they are then calculated within age classes. The aim of the MCO algorithm is, however, to minimize the overall $\Delta C$, i.e. within and between age classes, which is achieved if there is very little mixing of sublines (Robertson, 1964). Therefore, we included an extra restriction to restriction (3) on the distribution of parents over age classes, such that MCO would select not only animals from the oldest age class, but also one sire and one dam from the second-oldest age class. This is not the minimum possible mixing of sublines in a steady-state breeding scheme.

For these schemes with a restricted distribution of parents over age classes, the generation interval was 3.89 years for both RAND and MC mating schemes with 10 newborn animals per year (Table 3 ). The $\Delta F$ (0.0017 and 0.0018 per year for RAND and MC mating, respectively) and $\Delta C(0.0016$ and 0.0017 per year for RAND and MC mating, respectively) was similar for the two mating schemes. Note that $\Delta F$ was approximately $L$ times smaller for this scheme compared with the scheme with sublines (Table 1), because of the $L$ times larger population size.

In general, the two different mating schemes resulted in very similar breeding schemes: generation interval, number of selected animals and selection candidates were the same for both mating schemes. The correlation between genes within individuals, $\alpha$, and the variance of long-term contributions, $S_{\mathrm{c}}^{2}$, were somewhat lower for the MC scheme compared with the RAND scheme, but these differences where not large enough to affect predicted rates of inbreeding, $\Delta F_{\mathrm{p}}$. The $\alpha$ equalled -0.046 for RAND mating, which equals the expected value $-1 /(2 N-1)$ (Caballero, 1994), where $N$ is the number of selected animals per year.

The $S_{\mathrm{c}}^{2}$ and $\alpha$ were not given for the scheme with sublines (Table 1), because sublines did continuously drift further apart and thus $S_{\mathrm{c}}^{2}$ and $\alpha$ did not reach stable values. 
Table 3. Rate of inbreeding per year $(\Delta F)$, increase in coancestry per year $(\Delta C)$, variance of long-term contributions $\left(S_{\mathrm{c}}^{2}\right)$, correlation between genes within an individual $(\alpha)$, predicted $\Delta F\left(\Delta F_{\mathrm{p}}\right)$, generation interval of sires/dams $(L)$, number of selected sires/dams ( $N \mathrm{sel})$ and number of male/female selection candidates (Ncand) for schemes with 6, 10 and 20 newborn animals per year and 100\% survival, but with restriction on distribution of parents over age classes ${ }^{a}$

\begin{tabular}{|c|c|c|c|c|c|c|c|c|}
\hline Mating method ${ }^{b}$ & $\Delta F^{c}$ & $\Delta C^{c}$ & $S_{\mathrm{c}}^{2}$ & $\alpha$ & $\Delta F_{\mathrm{p}}{ }^{d}$ & $L$ (years) & $N$ sel & Ncand \\
\hline \multicolumn{9}{|l|}{ Six animals } \\
\hline RAND & $0 \cdot 0018$ & $0 \cdot 0028$ & 0.035 & -0.075 & 0.0031 & $3 \cdot 82 / 3 \cdot 82$ & $3 \cdot 9 / 4 \cdot 0$ & $12 / 12$ \\
\hline $\mathrm{MC}$ & $0 \cdot 0029$ & $0 \cdot 0030$ & $0 \cdot 033$ & $-0 \cdot 109$ & $0 \cdot 0032$ & $3 \cdot 83 / 3 \cdot 82$ & $3 \cdot 9 / 3 \cdot 9$ & $12 / 12$ \\
\hline \multicolumn{9}{|l|}{ Ten animals } \\
\hline RAND & $0 \cdot 0017$ & $0 \cdot 0016$ & $0 \cdot 084$ & $-0 \cdot 046$ & $0 \cdot 0018$ & $3 \cdot 89 / 3.89$ & $5 \cdot 9 / 5 \cdot 9$ & $20 / 20$ \\
\hline $\mathrm{MC}$ & $0 \cdot 0018$ & $0 \cdot 0017$ & $0 \cdot 073$ & -0.081 & $0 \cdot 0019$ & $3 \cdot 89 / 3.89$ & $5 \cdot 9 / 5 \cdot 9$ & $20 / 20$ \\
\hline \multicolumn{9}{|l|}{ Twenty animals } \\
\hline RAND & $0 \cdot 0006$ & $0 \cdot 0009$ & $0 \cdot 042$ & -0.024 & $0 \cdot 0012$ & $3 \cdot 94 / 3 \cdot 94$ & $10 \cdot 9 / 10 \cdot 8$ & $40 / 40$ \\
\hline $\mathrm{MC}$ & 0.0009 & 0.0009 & 0.039 & -0.051 & $0 \cdot 0012$ & $3.94 / 3.94$ & $10 \cdot 9 / 10 \cdot 8$ & $40 / 40$ \\
\hline
\end{tabular}

a At least one sire and one dam are from the second-oldest age class.

${ }^{b}$ RAND, random mating; MC, minimum coancestry mating.

${ }^{c}$ Average of years 96-100; standard errors are between 0.0001 and 0.0020.

${ }^{d} \Delta F_{\mathrm{p}}$ is predicted with (1) using observed $S_{\mathrm{c}}^{2}, N$ and $\alpha$.

Table 4. Rate of inbreeding per year $(\Delta F)$, increase in coancestry per year $(\Delta C)$, variance of long-term contributions $\left(S_{\mathrm{c}}^{2}\right)$, correlation between genes within an individual $(\alpha)$, predicted $\Delta F\left(\Delta F_{\mathrm{p}}\right)$, generation interval of sires/dams $(L)$, number of selected sires/dams ( $N \mathrm{sel})$ and number of male/female selection candidates (Ncand) for schemes with 6, 10 and 20 newborn animals per year and 70\% survival

\begin{tabular}{|c|c|c|c|c|c|c|c|c|}
\hline Mating method ${ }^{a}$ & $\Delta F^{b}$ & $\Delta C^{b}$ & $S_{\mathrm{c}}^{2}$ & $\alpha$ & $\Delta F_{\mathrm{p}}^{c}$ & $L$ (years) & $N$ sel & $N$ cand \\
\hline \multicolumn{9}{|l|}{ Six animals } \\
\hline RAND & $0 \cdot 0077$ & 0.0079 & $2 \cdot 71$ & -0.074 & $0 \cdot 0079$ & $4 \cdot 43 / 4 \cdot 44$ & $4 \cdot 1 / 4 \cdot 1$ & $9 \cdot 6 / 9 \cdot 5$ \\
\hline $\mathrm{MC}$ & $0 \cdot 0083$ & $0 \cdot 0078$ & $2 \cdot 80$ & $-0 \cdot 108$ & $0 \cdot 0082$ & $4 \cdot 46 / 4 \cdot 37$ & $4 \cdot 2 / 4 \cdot 3$ & $9 \cdot 6 / 9 \cdot 7$ \\
\hline \multicolumn{9}{|l|}{ Ten animals } \\
\hline RAND & $0 \cdot 0043$ & 0.0044 & $2 \cdot 65$ & $-0 \cdot 043$ & $0 \cdot 0046$ & $4 \cdot 39 / 4 \cdot 46$ & $7 \cdot 1 / 7 \cdot 1$ & $16 \cdot 0 / 15 \cdot 6$ \\
\hline $\mathrm{MC}$ & $0 \cdot 0046$ & $0 \cdot 0045$ & $2 \cdot 55$ & -0.074 & $0 \cdot 0045$ & $4 \cdot 50 / 4 \cdot 54$ & $7 \cdot 3 / 7 \cdot 1$ & $15 \cdot 7 / 16 \cdot 2$ \\
\hline \multicolumn{9}{|l|}{ Twenty animals } \\
\hline RAND & $0 \cdot 0021$ & $0 \cdot 0021$ & $2 \cdot 57$ & -0.020 & $0 \cdot 0021$ & $4 \cdot 60 / 4 \cdot 56$ & $14 \cdot 6 / 14 \cdot 6$ & $32 \cdot 5 / 32 \cdot 1$ \\
\hline $\mathrm{MC}$ & $0 \cdot 0022$ & 0.0022 & $2 \cdot 48$ & -0.044 & $0 \cdot 0022$ & $4 \cdot 56 / 4 \cdot 45$ & $14 \cdot 7 / 14 \cdot 6$ & $31 \cdot 6 / 32 \cdot 3$ \\
\hline
\end{tabular}

a RAND, random mating; MC, minimum coancestry mating.

${ }^{b}$ Average of years 96-100; standard errors are between 0.0001 and 0.0020.

${ }^{c} \Delta F_{\mathrm{p}}$ is predicted with (1) using observed $S_{\mathrm{c}}^{2}, N$ and $\alpha$.

\section{(ii) $70 \%$ survival}

Similar to schemes with $100 \%$ survival, $\Delta F$ was about the same for the MC mating (0.0046), and RAND mating (0.0043) for the schemes with $70 \%$ survival (Table 4). The $\alpha$ was low for the MC scheme (-0.074) compared with the RAND scheme $(-0.043), S_{\mathrm{c}}^{2}$ was about the same $(2.55$ and 2.65 for MC and RAND mating, respectively) and the predicted rate of inbreeding, $\Delta F_{p}$, was about the same for the two mating schemes.

For schemes with 6 and 20 newborn animals per year, results were similar to schemes with 10 newborn animals per year, except that the variation was larger for the small schemes with only three animals per sex, because of a larger sampling error (not shown) and the effect of culling, especially for RAND mating.

The results were compared with $\Delta F$ for populations where simply the oldest $(6,10$ and 20) animals were selected each year and there was random mating. The $\Delta C$ increased $31 \%, 42 \%$ and $39 \%$ for schemes with 6,10 and 20 newborn animals, respectively, for a survival rate of $50 \%$ compared with the $\mathrm{MCO}$ algorithm and random mating. For the survival rate of $70 \%, \Delta C$ was $41 \%, 36 \%$ and $52 \%$ higher and, finally, for the survival rate of $90 \%, \Delta C$ was $18 \%$, $31 \%$ and $50 \%$ higher for schemes with 6,10 and 20 newborn animals, respectively. We looked at $\Delta C$ instead of $\Delta F$ because of the smaller standard errors of $\Delta C$. There was no clear change of pattern of the 
superiority of the MCO schemes over the schemes that selected only the oldest animals when survival rate increased. This is possibly because a low survival rate results in most animals still being present in age class 1, i.e. a scheme with an almost discrete generation structure, whereas a very high survival rate (e.g. $100 \%$ ) also results in almost discrete generations. Intermediate survival rates result in schemes with different degrees of overlapping generations.

\section{Discussion}

\section{(i) Minimum coancestry selection}

A method has been presented that minimizes the rate of inbreeding for populations with overlapping generation structure and complex pedigrees. The algorithm operates at the level of coancestry and not inbreeding, because an initial increase in coancestry is more closely related to long-term inbreeding than the initial increase in inbreeding (Wray \& Goddard, 1994).

If survival rate $100 \%$, selection from only the oldest age class minimizes the $\Delta C$ across age classes, because $\Delta C$ is zero between age classes, and the population splits up into $L$ sublines separated by time, where $L$ is the generation interval. Rate of inbreeding within a subline will be $L$ times higher (Table 1) than the minimized rate of inbreeding that is achieved by the restriction that at least one sire and one dam comes from the second-oldest age class (Table 3). This restriction may seem somewhat ad hoc, but it should be noted that the animal that is selected from the second-oldest age class will generally be avoided for selection next year, because this animal already has offspring. The result is that the variance in family size is still small and $S_{\mathrm{c}}^{2}$ is only 0.033-0.084, implying that $\Delta F$ is only $3 \cdot 3-8.4 \%$ higher than its theoretical minimum value, which is achieved when $S_{\mathrm{c}}^{2}=0$. The later theoretical minimum can not be achieved, because the mixing of sublines results in $S_{\mathrm{c}}^{2}>0$.

For practical schemes with survival rates less than $100 \%$, the oldest age classes contain too few animals and some younger animals have to be selected. The algorithm then weights the selection of more and consequently younger animals against the reduction of the generation interval, accounting for the pedigree of available animals. A simple scheme, where only the oldest animals were selected, resulted in $18-52 \%$ higher $\Delta C$ and thus $\Delta F$ than the MCO scheme.

\section{(ii) MC mating}

It is expected that in the short term, inbreeding levels would decrease, but that in the long term, $\Delta F$ would increase for MC mating compared with RAND mating (Kimura \& Crow, 1963; Robertson, 1964; Ballou \&

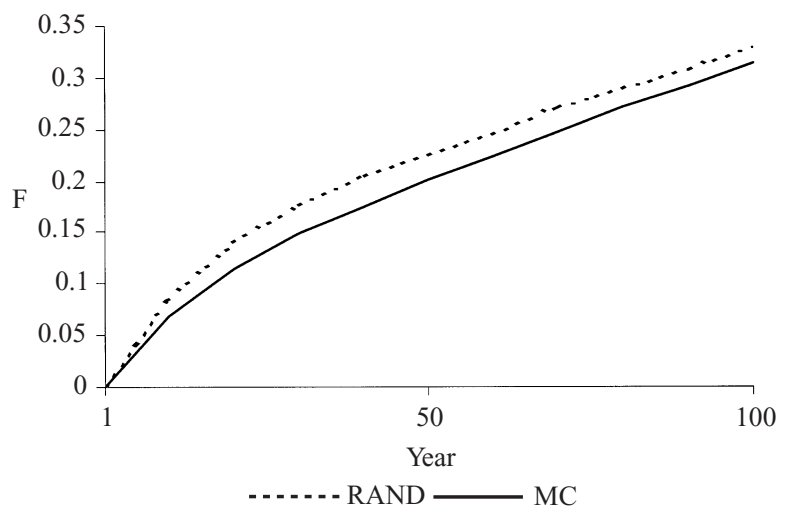

Fig. 1. Inbreeding $(F)$ for schemes with 6 newborn animals per year and random (RAND) or minimum coancestry (MC) mating and $100 \%$ survival.

Lacy, 1995; Wang, $1997 b$; and others). The shortand especially long-term effect of MC mating on inbreeding was, however, small here: the inbreeding level was lower for the MC than for the RAND scheme during all the 100 years (Fig. 1), but the inbreeding of $\mathrm{MC}$ was catching up with the inbreeding of RAND, resulting in a slightly higher $\Delta F$ for MC (Table 3). At around year 300 (results not shown), when the inbreeding was relatively high, the graphs with inbreeding values in Fig. 1 crossed over such that MC mating had marginally higher inbreeding values than RAND mating. Thus, although $\Delta F$ was slightly higher for MC mating, the actual inbreeding levels started lower for MC mating, caught up only at year 300 and were thereafter slightly higher.

\section{(iii) Practical schemes}

The different mating strategies hardly affected $\Delta F$, but because the level of inbreeding was lower for a long time period (more than 100 years), MC mating is preferred over RAND mating for the present schemes. For schemes with, for example, short generation intervals, the inbreeding levels of MC and RAND mating might become similar earlier than in Fig. 1, which makes inbreeding levels of MC and RAND mating schemes even more equal.

The schemes presented require full control of the family size and matings of animals. In practice, this may not be possible and the optimum contributions, c, can only be aimed at. Under these circumstances, the MCO algorithm seems most useful, because it adapts to the pedigree of the available animals (assuming the pedigree is known). For instance, if one family becomes too large by chance, its members will get lower contributions, and vice versa. However, $\Delta F$ may be substantially higher than in Tables 3 and 4, because of the reduced control over family size. Similarly, the MCO algorithm will adapt to possible distortions of the sex ratio of the populations. In 
situations where the population increases in size, MCO is expected to optimize selection across age classes, where it balances selection from younger age classes that contain more animals, against selection from old age classes that contain few animals.

When a population is geographically split up into several subpopulations, there are other factors that have to be considered, such as transportation difficulties and costs and social structure of the populations. This study does not consider these factors, but the subpopulations can be seen as the sublines of Table 1 that can easily become highly related when there is too little mixing.

\section{(iv) Conclusions}

1. With overlapping generations and $100 \%$ survival, average coancestry across the whole population can be minimized by breeding only from the oldest age class. This splits the population into sublines defined by age and increases inbreeding within sublines. The MCO algorithm does result in complete sublining with $100 \%$ survival.

2. With a restriction on the distribution of parents over age classes, such that not only animals from the oldest age class but also one animal from the secondoldest age class were selected, $\Delta F$ became $L$ times smaller than for the schemes with sublines, because the population was $L$ times larger.

3. If survival is less than $100 \%$, the algorithm weights the selection of more and consequently younger animals against the reduction of the generation interval, accounting for the pedigree of the available animals.

4. Based on these results, MCO and MC mating would be desirable for practical populations, because this resulted in lower levels of inbreeding for more than 100 years. Thereafter, inbreeding levels of RAND and $\mathrm{MC}$ mating were very similar. The $\Delta F$ was, however, somewhat higher for the MC mating schemes, because the inbreeding levels of the MC mating schemes caught up with the RAND schemes.

5. A scheme where only the oldest animals are selected led to an $18-52 \%$ higher $\Delta C$, and thus $\Delta F$, than MCO selection.

\section{Appendix}

\section{Calculation of optimum contributions}

The average relationship in equation (2) will be minimized under the restriction of the genetic contributions per sex in equation (3). For the optimization algorithm, a Lagrangian multiplier, $\lambda$, is used as a cost factor on the genetic contributions per sex and is calculated such that the contribution per sex actually becomes $0 \cdot 5$. The Lagrangian function to be optimized, $H$, is

$H=r_{1}^{2} \mathbf{c}^{\prime} \mathbf{A} \mathbf{c}+2 r_{1} \mathbf{c}^{\prime} \mathbf{A} \mathbf{J} \mathbf{r}_{2}+\mathbf{r}_{2}^{\prime} \overline{\mathbf{A}}_{22} \mathbf{r}_{2}-\left(\mathbf{c}^{\prime} \mathbf{Q}-\mathbf{s}^{\prime}\right) \lambda$.

Equating to zero the derivative of $H$ with respect to the genetic contributions of the newborn progeny, $\mathbf{c}$, yields

$2 r_{1}^{2} \mathbf{A c}+2 r_{1} \mathbf{A J r}_{2}-\mathbf{Q} \lambda=0$.

Solving for $\mathbf{c}$ yields

$\mathbf{c}=\mathbf{A}^{-1} \mathbf{Q} \lambda / 2 r_{1}^{2}-\mathbf{J r}_{2} r_{1}$.

Equating to zero the derivative of $H$ with respect to the cost factor on the contribution per sex, $\lambda$, yields

$s^{\prime}=\mathbf{c}^{\prime} \mathbf{Q}$.

Substituting the solutions for $\mathbf{c}$ into (A3) and solving for $\lambda$, gives

$\lambda=2 r_{1}\left(\mathbf{Q}^{\prime} \mathbf{A}^{-1} \mathbf{Q}\right)^{-1}\left(r_{1} \mathbf{S}+\mathbf{Q}^{\prime} \mathbf{J} \mathbf{r}_{2}\right)$.

Finally, the solution for $\lambda$ in (A4) is substituted into (A2) in order to get $\mathbf{c}$ for each selection candidate.

We are grateful to Mike Goddard, Piter Bijma, Pim Brascamp, Ab Groen, Kor Oldenbroek and an anonymous referee for helpful comments and suggestions.

\section{References}

Ballou, J. D. \& Lacy, R. C. (1995). Identifying genetically important animals for management of genetic variation in pedigreed population. In Population Management for Survival and Recovery: Analytical Methods and Strategies in Small Population Conservation (ed. J. D. Ballou, M. Gilpin \& T. J. Foose), pp. 76-111. New York: Columbia University Press.

Bijma, P. \& Woolliams, J. A. (1999). Prediction of genetic contributions and generation intervals in populations with overlapping generations under selection. Genetics 151, 1197-1210.

Caballero, A. (1994). Developments in the prediction of effective population size. Heredity 73, 657-679.

Caballero, A. \& Toro, M. A. (2000). Interrelations between effective population size and other pedigree tools for the management of conserved populations. Genetical Research 75, 331-343.

Grundy, B., Villanueva, B. \& Woolliams, J. A. (1998). Dynamic selection procedures for constrained inbreeding and their consequences for pedigree development. Genetical Research 72, 159-168.

Hill, W. G. (1974). Prediction and evaluation of response to selection with overlapping generations. Animal Production 18, 117-139.

Kimura, M. \& Crow, J. F. (1963). On the maximum avoidance of inbreeding. Genetical Research 4, 399-415.

Meuwissen, T. H. E. (1997). Maximising the response of selection with a predefined rate of inbreeding. Journal of Animal Science 75, 934-940.

Meuwissen, T. H. E. \& Sonesson, A. K. (1998). Maximizing the response of selection with a predefined rate of inbreeding: overlapping generations. Journal of Animal Science 76, 2575-2583. 
Press, W. H., Flannery, B. P., Teukolsky, S. A. \& Vetterling, W. T. (1989). Minimization or maximization of functions. In Numerical Recipes: The Art of Scientific Computing. Cambridge: Cambridge University Press.

Robertson, A. (1964). The effect of non-random mating within inbred lines on the rate of inbreeding. Genetical Research 5, 164-167.

Sonesson, A. K. \& Meuwissen, T. H. E. (2000). Mating schemes for optimum contribution selection with constrained rates of inbreeding. Genetics, Selection, Evolution 32, 231-248.

Sonesson, A. K., Grundy, B., Woolliams, J. A. \& Meuwissen, T. H. E. (2000). Selection with control of inbreeding in populations with overlapping generations: a comparison of methods. Animal Science 70, 1-8.

Wang, J. L. (1997a). More efficient breeding systems for controlling inbreeding and effective size in animal populations. Heredity 79, 591-599.

Wang, J. L. (1997b). Effect of excluding sib matings on inbreeding coefficient and effective size of finite populations. Biometrics 53, 1354-1365.

Wang, J. \& Hill, W. G. (2000). Marker-assisted selection to increase effective population size by reducing mendelian segregation variance. Genetics 154, 475-489.

Wang, J. L., Xu, J. C., Song, J. Z. \& Tang, W. S. (1994). Study on mating systems for controlling inbreeding levels in domestic animal populations with overlapping generations. In Proceedings of the Fifth World Congress on Genetics Applied to Livestock Production 21, 536-539.

Woolliams, J. A.\& Thompson, R. (1994). A theory of genetic contributions. In Proceedings of the Fifth World Congress on Genetics Applied to Livestock Production 19, 127-134.

Wright, S. (1921). Systems of mating. Genetics 6, 111-178.

Wright, S. (1931). Evolution in mendelian populations. Genetics 16, 97-159.

Wright, S. (1969). Evolution and the Genetics of Populations, vol. 2, The Theory of Gene Frequencies. Chicago: University of Chicago Press.

Wray, N. R. \& Goddard, M. E. (1994). Increasing longterm response to selection. Genetics, Selection, Evolution 26, 431-451.

Wray, N. R. \& Thompson, R. (1990). Prediction of rates of inbreeding in selected populations. Genetical Research 55, $41-54$. 